\title{
Planetary Exploration; Mars on the Scope
}

\author{
Buenestado $\mathrm{JF}^{1}$, Zorzano $\mathrm{MP}^{2}$, Salinas $\mathrm{AS}^{3}$, Méndez $\mathrm{CF}^{3}$ and Martín-Torres $\mathrm{J}^{1,4^{*}}$ \\ ${ }^{1}$ Division of Space Technology, Department of Computer Science, Electrical and Space Engineering, Luleå Technical University, Kiruna, Sweden \\ ${ }^{2}$ Centro de Astrobiología (INTA-CSIC), Torrejón de Ardoz, Madrid, Spain \\ ${ }^{3}$ Escuela de Ingeniería Aeronáutica y del Espacio, Universidad Politécnica, Madrid, Spain \\ ${ }^{4}$ Instituto Andaluz de Ciencias dela Tierra (CSIC-UGR), Granada, Spain
}

*Corresponding author: Martín-Torres J, Division of Space Technology, Department of Computer Science, Electrical and Space Engineering, Luleå Technical University, Kiruna, Sweden, Tel: +46 (0)980 67545; E-mail: javier.martin-torres@ltu.se

Rec date: June 09, 2015; Acc date: July 02, 2015; Pub date: July 06, 2015

Copyright: (c) 2015 Buenestado JF, et al. This is an open-access article distributed under the terms of the Creative Commons Attribution License, which permits unrestricted use, distribution, and reproduction in any medium, provided the original author and source are credited.

\begin{abstract}
This article summarizes a practical case of introduction to research and planetary exploration through the analysis of data from the Rover Environmental Monitoring Station (REMS), one of the ten scientific instruments on board the Curiosity rover of the Mars Science Laboratory (MSL), currently operating at the impact crater Gale, on Mars. It is the main aim of this work to show how the data that are publicly available at the Planetary Data System (PDS) can be used to introduce undergraduate students and the general public into the subject of surface exploration and the environment of Mars. In particular, the goal of this practice was to investigate and quantify the heat-flux between the rover spacecraft and the Martian surface, the role of the atmosphere in this interaction, and its dependence with seasons, as well as to estimate the thermal contamination of the Martian ground produced by the rover. The ground temperature sensor (GTS) of the REMS instrument has measured in-situ, for the first time ever, the diurnal and seasonal variation of the temperature of the surface on Mars along the rover traverse. This novel study shows that the rover radiative heat flux varies between 10 and $22 \mathrm{~W} / \mathrm{m}^{2}$ during the Martian year, which is more than $10 \%$ of the solar daily averaged insolation at the top of the atmosphere. In addition, it is shown that the radiative heat flux from the rover to the ground varies with the atmospheric dust load, being the mean annual amplitude of the diurnal variation of the surface temperature of $76 \mathrm{~K}$, as a result of solar heating during the day and infrared cooling during the night. As a remarkable and unexpected outcome, it has been established that the thermal contamination produced by the rover alone induces, on average, a systematic shift of $7.5 \mathrm{~K}$, which is indeed about $10 \%$ of the one produced by solar heating. This result may have implications for the design and operation of future surface exploration probes such as Insight.
\end{abstract}

Keywords: Martian; Planetary exploration; Astronomical observations; Planetary missions; Crater Gale; Solar radiation; Radiative

\section{Introduction}

Exploration is the act of searching for the purpose of discovery of information or resources. With the invention of rockets in the 20th century, humanity has continued its impulse to explore by moving beyond Earth, having travelled to the Moon and sent robotic explorers to other planets such as Mars. As the American astrophysicist Neil de Grasse said in his testimony to the U.S. Senate, "Exploration of the unknown might not strike everyone as a priority. Yet audacious visions have the power to alter mind-state, to change assumptions of what is possible. When a nation permits itself to dream big, those dreams pervade its citizens' ambitions" (Past, Present, and Future of NASA, U.S. Senate Testimony, Neil de Grasse Tyson, March 7, 2012).

Planetary exploration is a relatively new field of research included in space sciences which is becoming more and more active and wider at an accelerated pace. The almost daily discovery of new planets in far star systems by means of remote sensing instruments opens new paths for future exploration that will start to be traversed through the exploration of our system. The exploration of Mars, in particular, will be in many senses the first step in facing this challenge since, indeed, we live in what can be said to be the Golden Age of Mars exploration.
It is close to the Earth, it is a rocky planet, and its surface environment, although harsh, is not as inhospitable as that of Venus, making it the most accessible planet in our system. The enrichment of our knowledge of Mars will lead to a better understanding of Mars itself, the historical evolution of our solar system, and the evolution and dynamics of the Earth as a part of it, as well as to the improvement of the technology available for the missions to come.

Yet, the exploration of another planet requires the use of dedicated spacecraft designed, launched and operated in the context of large international collaborations, including space agencies, universities, research centers and industrial partners. Parts of the legacy of these missions are the scientific discoveries and novel technological applications that are developed to overcome new challenges. Currently there are five orbiters observing Mars, namely, Mars Odyssey, Mars Express, Mars Reconnaissance Orbiter, Mars Atmosphere and Volatile Evolution (MAVEN), and Mars Orbiter Mission (MOM) (listed by date of arrival), as well as two robotic rovers: Opportunity, which has been working for more than a decade now, and Curiosity, operating within the crater Gale, near the Martian equator, since august 2012. All these probes are equipped with very efficient instruments and have been, in some cases, far more successful than expected. This fact has permitted the collection of a huge amount of high quality raw data that have to be converted into useful information through a thoughtful analytic work, exceeding the capability of the research teams involved in some cases. On the other hand, although the accuracy reached by 
these instruments is a clear advantage, it also poses the problem of making the extraction of reliable information more and more complex. For all the aforementioned, it is convenient to implement strategies to extend the analytical work beyond the research teams involved, looking for support wherever it can be given. This is one of the aims that inspired the creation of the Planetary Data System (PDS), an archive in which data from all the planetary missions, astronomical observations, and laboratory measurements performed by NASA up to date are stored for public access. As the agency states, it is designed, among other things, "to enable future scientists who are unfamiliar with the original experiments to analyze the data". The PDS archives and distributes scientific data from NASA planetary missions with the purpose of ensuring the long-term usability of NASA data and to stimulate advanced research, making possible for scientific teams all over the world to make contributions to the research in planetary exploration, and favoring this way the continuous revision of the data

The basic goal of this article is to introduce the reader to the use of Mars exploration data that are publicly available at the PDS, and to provide a practical case which can be used both for outreach purposes and more advanced planetary exploration research. In this sense, this article expects to serve as a user's guide for those scientists involved in education who, somehow, could find interesting to reproduce a similar practice. On a second stage, the practice can be adapted easily to lower educational levels, serving this way to spread this particular area of science among a broader public, from the conviction that outreach is an unique tool to make society understand the importance of developing this field within the next years, as well as a way to share part of the products that this large technical, scientific and economical international effort entails.

\section{Curiosity and the REMS environmental station}

On August the 5th, 2012, the Mars Science Laboratory (MSL) rover, as well known as Curiosity, landed at $6^{\circ} \mathrm{S}, 137.4^{\circ} \mathrm{E}$, in the NW part of the crater Gale, a $154 \mathrm{~km}$ wide impact crater allocated near the foot of a steep section of the dichotomy boundary, whose floor is at $4.5 \mathrm{~km}$ below datum. After more than 1,000 Earth days (one and a half Martian years) of surface exploration at the floor of the crater, the Rover Environmental Monitoring Station (REMS) on board the Curiosity rover $[1,2]$ has acquired an unprecedented record of pressure, air and ground temperatures, surface ultraviolet radiation (in the range $200 \mathrm{~nm}-400 \mathrm{~nm}$ ), and relative humidity from another planet along its more than $10.5 \mathrm{~km}$ long traverse. Only during its one Martian year nominal mission, REMS has recorded more than 15 million of measurements, far more than originally anticipated, and it has provided the first in-situ observations of the diurnal and seasonal evolution of those parameters [2-4]. REMS in-situ meteorological observations are the first of its kind from the South hemisphere, at a site with complex topography in the Martian tropics, what provides a unique opportunity to sample an environment that is different from any previous one $[5,6]$. Every year of MSL operation is very productive and demanding for all the instrument teams. For instance, and only during 2014, REMS has been actively operating 238 days, within which the REMS sensors have provided 9,233,755 acquisitions. The nominal cadence of REMS measurements is defined as hourly five-minute acquisitions at $1-\mathrm{Hz}$ frequency, including night-time. Additionally, it is typical to sample between three and seven extended one-hour blocks every sol (a Martian day), also at 1-Hz frequency. The REMS station consists of two booms mounted on the mast of the rover at $1.6 \mathrm{~m}$ height above the ground, separated with an angle of $120^{\circ}$, together with two extra independent units that are embedded within the rover deck and house the Ultraviolet Sensor (UVS), the Pressure Sensor (PS) and the ICU (Instrument Control Unit), (Figure 1).

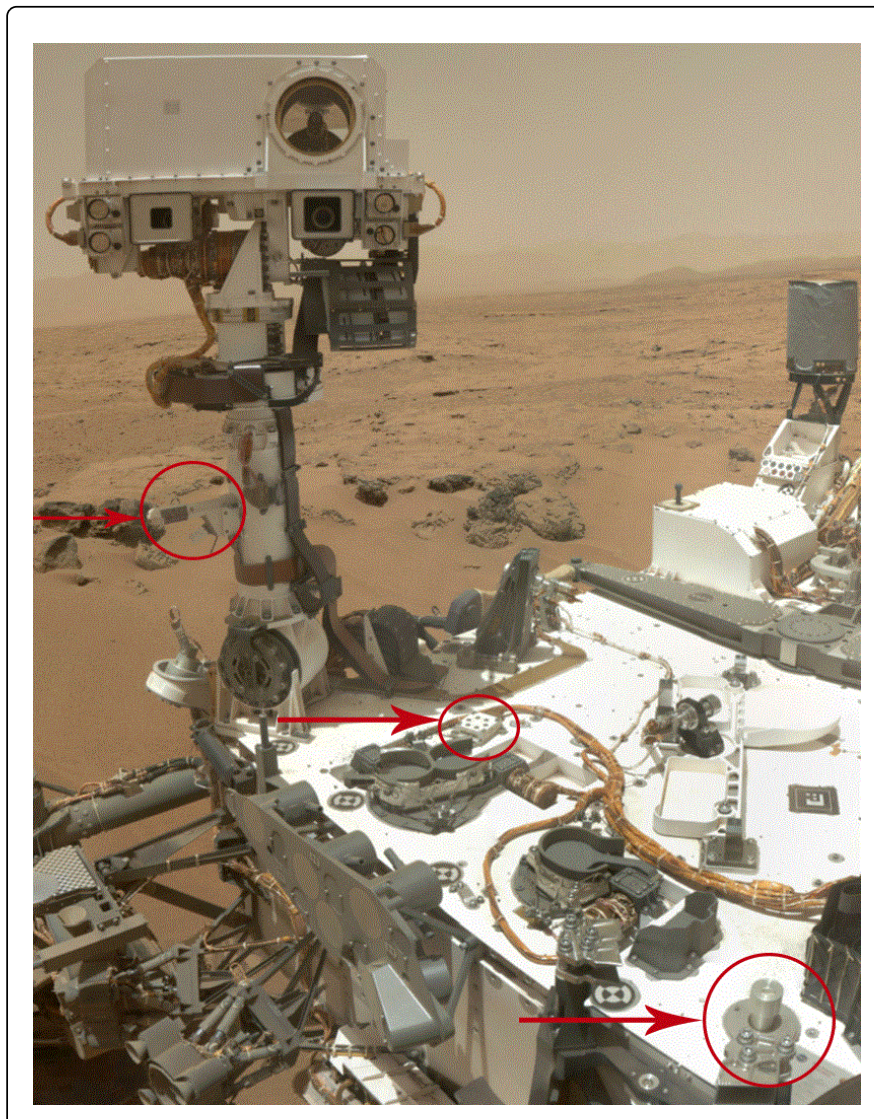

Figure 1: Situation of REMS's sensors on Curiosity. The picture shows, from top to bottom, boom 2 (with WS, ATS, and HS) and, upon the rover deck, UVS and PS. The shadow of the mast, which can be seen close to UVS, cover this latter in some moments of the day disrupting its measurements, a circumstance that must be taken into account when processing the data it provides. Boom 1 (WS, ATS and GTS) is hidden behind the rover mast, pointing to the side of the rover. Credit NASA/JPL-Caltech/MSSS.

Each boom has a Wind Sensor (WS) and an Air Temperature Sensor (ATS). One of them (boom 1) has also a Ground Temperature Sensor (GTS) and the other one (boom 2), a Humidity Sensor (HS). Data gathered by all these sensors are then checked, labeled, documented and formatted for their public release to the PDS providing an everlasting and invaluable legacy for future research and educational purposes. Those analyzed in this work correspond to observations in the period 2012-2014, and are publicly available and documented at the NASA's PDS [Planetary Atmosphere Node at http:// atmos.nmsu.edu/PDS/data/].

Regarding the topic treated in this article, it is important to remark that the GTS has measured, for the first time ever, the diurnal and seasonal variation of the surface temperature within a footprint of about $100 \mathrm{~m}^{2}$ along the rover traverse, providing a huge amount of valuable data in order to unveil the near surface thermal environment of the regolith within Gale crater [2,7]. 
Yet, the raw data that REMS sends are not directly useful because they are taken from a platform that introduces perturbations in the environment to be monitored, so it is necessary to estimate accurately the magnitude of such perturbations to correct them reliably. As examples of the factors that affect the observations of the environmental sensors can be mentioned the daily activity of the rover, the differences between the traverse and stop acquisitions, the effects of shadows cast by the mast or the robotic arm, the heating of the metallic structure of the rover by solar radiation, the permanent protective heating of the electronic bay within the rover deck, the interaction of winds with the spacecraft, the thin-film of air that is warmer in the vicinity of the rover, etc. However, one of the most important perturbations is the heating of the spacecraft and its environment by the electrical power supply of Curiosity, the Radioisotope Thermoelectric Generator (RTG). It works by converting heat from the natural decay of radioisotope materials into electricity, containing a total of 4.8 kilograms of plutonium dioxide (including $\mathrm{Pu}-238$ ) that initially provides approximately 2,000 watts of thermal power and 110 watts of electrical power when exposed to deep space environments. The hottest temperatures recorded on the rover occur at the RTG $\left(\mathrm{T}=198^{\circ} \mathrm{C}=471 \mathrm{~K}\right)$, making this point the hottest object on Mars, and radiating energy to the environment. In the cold temperature regime part of the RTG, heat is used to maintain warm the rover electronics and scientific instruments located in the rover deck bay. An active fluid loop heat rejection system (HRS) radiatively collects waste heat from the RTG to maintain the science and avionics payload boxes on the rover deck bay above their minimum allowable flight temperatures [8]. On the contrary, when the rover deck bay is too hot, the HRS rejects heat to the environment via the 'cold plates' and top deck radiators to maintain safe interface temperatures [8]. This system-wide thermal control does not draw on the rover's electrical power, precludes the need for radioisotope heater units for spot heating, and keeps the rover systematically warmer than the ambient.

It is worth to mention that the dimensions of the Curiosity Rover are large, with a length of 3 meters (not counting the arm) and a width of 2.8 meters. If the height of the rover body is taken as 0.5 meter, this gives a total rover body surface of $22.6 \mathrm{~m}^{2}$. Given that the RTG, according to specifications, releases approximately 2,000 $\mathrm{W}$ of thermal power to deep space environments, we foresee an average dissipation of $88.5 \mathrm{~W} / \mathrm{m}^{2}$ from this spacecraft. This is a large energy flux, whose effect should be detectable at the Martian surface.

\section{Materials and Methods}

REMS data are allocated in PDS's files in packs of 90 sols uploaded with a delay or 90 days. These data go through a preliminary treatment before their publication, but they include electronic noise measurements, so they can be considered as "raw" data, from which useful information must be extracted in different levels of analysis. The files include information about the circumstances in which data were taken, such as confidence level, state of the electronics, dust deposition on the UVS's photodiodes (Figure 2), recalibration quality, shadow over UVS or GTS field of view, etcetera.

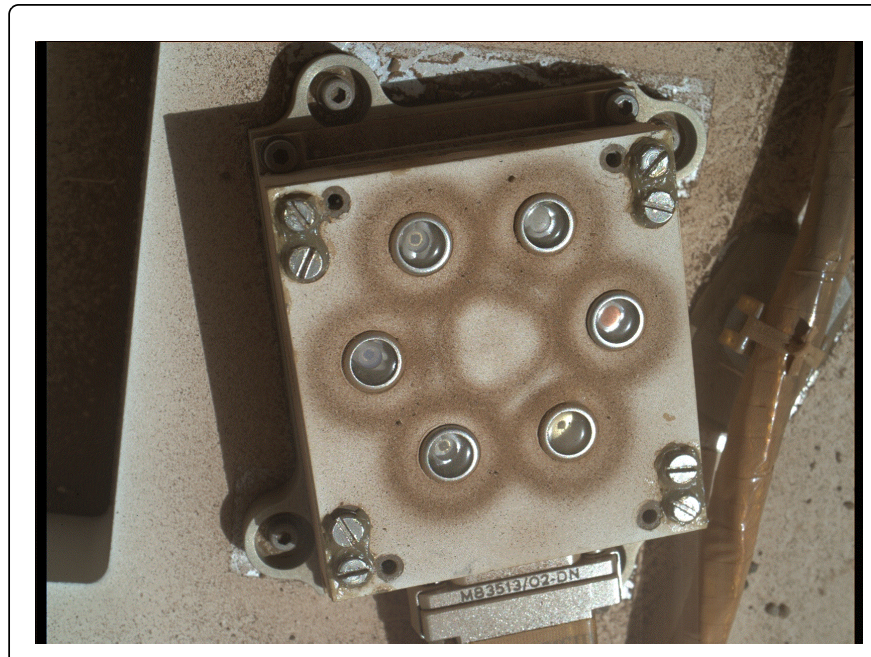

Figure 2: Dust coverage on UVS on sol 526. Each photodiode is surrounded by a magnetic ring to deviate the dust particles avoiding the deposition of dust on the field of view of the sensors. There is a seventh magnetic ring in the centre that delimitates an area used as a reference to estimate the amount of dust deposited in order to calibrate the measurements taken. Although the expected period of utility of UVS was only a few months at first, the magnets solution has proved to be very effective, and UVS keeps on functioning actually. Credit NASA/JPL-Caltech/MSSS.

The plots have been performed by means of the open source plotting program gnu plot (http://www.gnuplot.info/), which is particularly useful in the treatment of large data file as it is the case of the daily raw acquisitions of REMS. Data processing has been performed using specific codes programmed for this practice in fortran95, also an open source programming language, as well as with the use of the routines splint and spline from the book Numerical Recipes in Fortran [9], which implements the application of wellknown mathematical methods for the resolution of practical problems in modular routines programmed in Fortran. In particular, these routines are used to perform the cubic interpolation of a function within an interval where a set of known tabulated values exists. REMS acquisitions take place usually within the first 5 minutes of every hour, and occasionally during extended acquisitions of one hour within different intervals for each sol. The use of these routines allows to interpolate the "expected" observables at times when there are no real acquisitions and thus to compare different sols with different observational cadences. The REMS ENVRDR data set in the PDS contains partially processed REMS data converted from electrical to environmental magnitudes with only few corrections (calibration products and ancillary information). Then the MODRDR data set includes further treatment of the REMS data to correct from some factors. Data are a time ordered sequence of rows organized into a table, taken at a maximum resolution of one second, and each data product contains one sol of activity and has information from all sensors. Columns with related information are placed together in the following order: time stamp and the corresponding LMST (Local Mean Standard Time) in hours, minutes, and seconds in which the measurements were taken, and then WS, GTS, ATS, UVS, HS, and PS data. The labels of each column can be seen in the ENVRDR5.FMT and ENVRDR5.FMT files. 


\section{Practical study case}

The goal of the practice, that has been performed during three months within an aerospace engineering internship course, is to train students in the investigation of the present day environmental conditions on the surface of Mars through data gathered by REMS discerning, by using some of the tools and techniques that are used to face this kind of analytic and scientific work, the perturbation that the platform (the rover itself) introduces in several measured parameters.

This practice had three major objectives:

- To estimate the radiative heat-interchange between the MSL platform and the Martian surface.

- To investigate its variation along the year comparing its magnitude with the solar radiation.

- To quantify the influence of this heat-interchange produced by the presence of the spacecraft on the Martian ground temperature.

In order to develop the basic skills for the use of the computer tools to perform the analysis, the practice started with a preliminary study of the radiative thermal contamination of the ground by the RTG using the program gnu plot to plot initially raw data from the PDS. This first exercise permitted to appreciate the usual artifacts produced by the rover instrument noise, and air thermal fluctuations around the hot platform, or the effect of self-heating of the HS electronics on his relative humidity measurements (Figure 3 ).

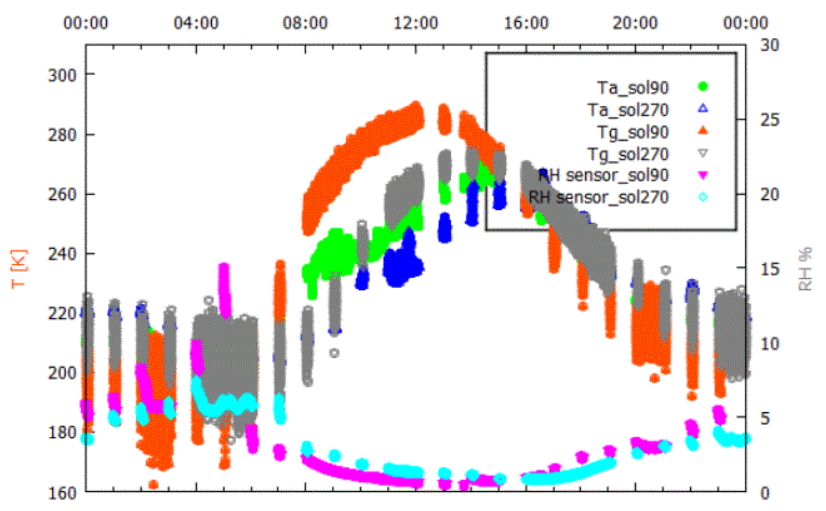

Figure 3: First plot of air and ground temperature and relative humidity for sols 90 and 270. It has been performed with GNUPLOT to illustrate how the multiple fluctuation sources impede to extract any useful information, making necessary further data treatments in order to develop the appropriate analysis.

In Figure 3 the data are shown as a function of LMST. Among other things, it showed the noisy signal of the ground temperature sensor measurements, which is more pronounced at night, when the real values are hidden in noise measurements that range up to $40 \mathrm{~K}$. The data processing was performed by developing a fortran 95 program that averages consecutive data-sets within a period of 5 minutes, in the case of nominal acquisitions, and over intervals of 15 minutes in the extended ( 1 hour) acquisitions. Furthermore, if within the sequential analysis of REMS observations a gap of 2 minutes or more is detected, a new averaging sequence is started. This program evaluates then several physical magnitudes derived from these observables, such as the radiative heat-flux, averaged over one sol. The results of the 5 and 15 minutes averages are shown in Figure 4.

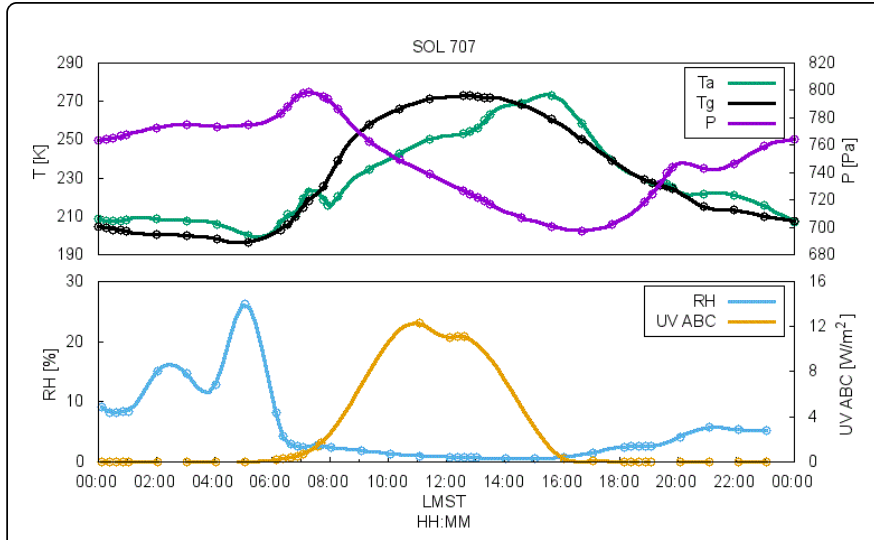

Figure 4: Data after average of 5 minutes. The plots show the daily evolution of several parameters monitored by REMS for sol 707, namely, air and ground temperature, pressure, relative humidity and UV radiation. They show the 5-minutes-averages of REMS data.

Next we evaluated the radiative heat flux between the rover platform and the ground. In order to have a temperature that represents that of the metallic structure of the rover, we used the temperature of the UVS, which is embedded within the rover deck. The radiative flux between two elements can be calculated using the Stefan-Boltzmann equation. Then:

$$
\mathrm{q}=\sigma \cdot \varepsilon 1 \cdot \varepsilon 2 \cdot \mathrm{F}(1 \rightarrow 2) \cdot(\operatorname{Tr} 4-\operatorname{Tg} 4)
$$

Where $\mathrm{q}$ is the radiative flux in $\mathrm{W} / \mathrm{m}^{2}, \sigma$ is the Stefan-Boltzmann constant $\left(5.6704 \cdot 10-^{8} \mathrm{~W} \cdot \mathrm{m}^{-}{ }^{2} \cdot \mathrm{K}-{ }^{4}\right), \mathrm{Tr}$ is the rover temperature in $\mathrm{K}$, and $\mathrm{Tg}$ is the brightness temperature of the ground in $\mathrm{K}$. $\varepsilon 1$ is the rover surface emissivity, which is determined by the properties of the white paint that covers it. We shall take $\varepsilon 1=0.88$, which is the typical infra-red (IR) emissivity of some space qualified paints; $\varepsilon 2$ is the ground surface emissivity, which for the IR range and the site explored by Curiosity can be assumed to be $\varepsilon 2=0.9$. In radiative heat transfer, a view factor $\mathrm{F}_{(1 \rightarrow 2)}$, is the proportion of the radiation from surface 1 that strikes surface 2 . The view factor of the rover side to the ground can be approximated by that of a vertical wall to the ground, which is $\mathrm{F}$ $(1 \rightarrow 2)=0.5$. The remaining energy is radiated to the sky and air.

The results are shown in Figures $5 \mathrm{a}$ and $5 \mathrm{~b}$ using the 5 minutes averages of the magnitudes $\mathrm{Tr}$ and $\mathrm{Tg}$. For comparison the measured incident UV irradiance is taken as a proxy for the full incident solar irradiance. Notice that in Figure 5a, the rover temperature is higher than that of the ground all along the day, with the exception of a few hours around local noon, when they are very similar. This is because during the central hours of the day the air temperature is much lower than the temperature of the ground. Convection at the boundary layer is active and this results in a natural cooling of the rover surface, whose temperature is reduced almost to that of the ground. Thus, the radiative heat-flux from the rover to the ground is always positive and can reach values as high as $22 \mathrm{~W} / \mathrm{m}^{2}$. In the example of Figure $5 \mathrm{~b}$, there is an anomaly in this thermal profile: there were shadows cast by the mast over the location of the UV sensors, and thus the local temperature of the platform was lower than that of other illuminated areas, including the ground. This results in an apparent negative radiative heat-flux, which is in fact only an artifact of this transient shadow. For this sol also, maximal heat-fluxes reach values of about 
Page 5 of 8

between $17 \mathrm{~W} / \mathrm{m}^{2}$ and $22 \mathrm{~W} / \mathrm{m}^{2}$. This energy flow is large and thus we foresee that this shall have a detectable effect on the local temperature of the ground at the site where the rover is located [10].

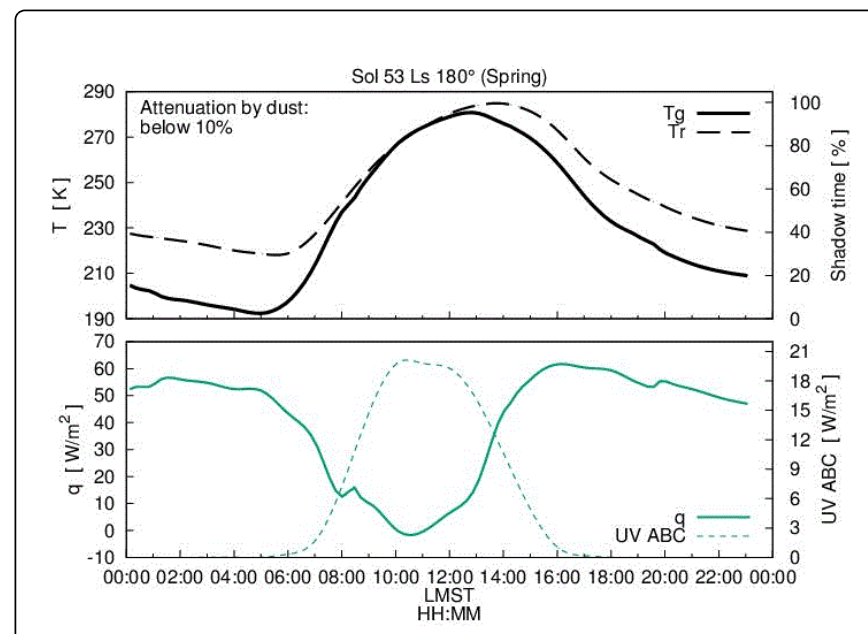

Figure 5a: Evolution of ground and rover temperature and radiation flux (q). The rover temperature $\operatorname{Tr}$ is taken from the sensor that monitors the temperature of the UVS.

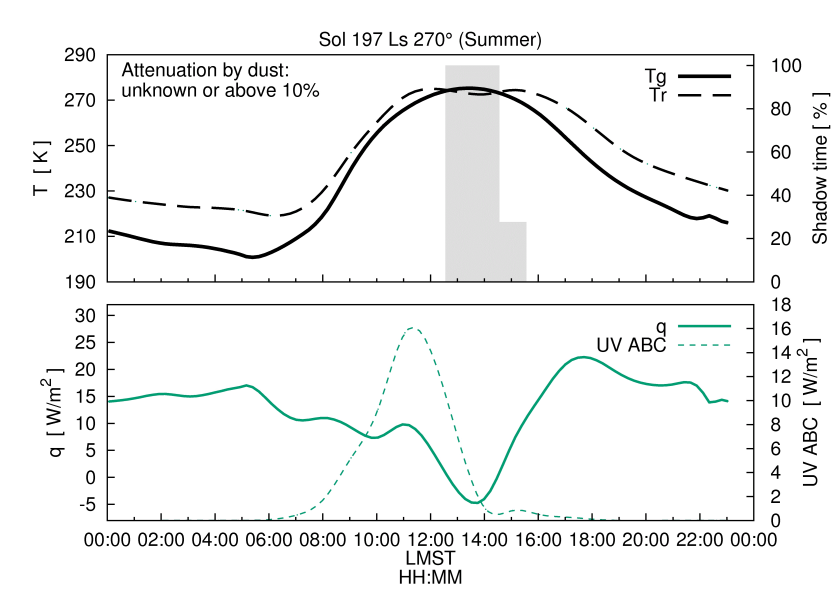

Figure 5b: Evolution of ground and rover temperature and radiation flux (q). Figure $5 \mathrm{~b}$ shows how when the sensor is covered by the shadow of the mast (grey area) this temperature drops.

Next, this analysis is performed for all the sols during one Martian year. The daily average of the heat-flux is calculated and shown as a function of sol. The incident daily insolation at the Top of the Atmosphere (TOA) is calculated for comparison using
Where $\mathrm{S}_{\mathrm{av}}$ is the solar insolation at a distance of $\mathrm{r}_{\mathrm{av}}=1.52 \mathrm{AU}$, which is the mean Mars-Sun distance in Astronomic Units, in $\mathrm{W} \cdot \mathrm{m}^{-2}$ (with $\mathrm{SO}=590 \mathrm{~W} \cdot \mathrm{m}^{-2}$ ) for a given latitude $\theta$ (for the landing site of MSL $\left.\theta=-4.5^{\circ}\right), \delta$ is the solar declination and depends on the inclination angle of Mars rotation axis and of Ls. $r_{\text {avr }}$, which is the relative orbital radius depending in turn on the orbit eccentricity $e=0.0934$ and on the difference of the solar angle Ls and the angle of reference at perihelium $\mathrm{Lsp}=250^{\circ} ; \varepsilon=25.2$ is the obliquity of Mars, and finally, $\mathrm{H}$ is the hour angle at sunset.

These results are compared with the atmospheric opacity which is a measurement of the amount of dust in the atmosphere and varies along the course of the mission. These magnitudes are shown in Figure 6.

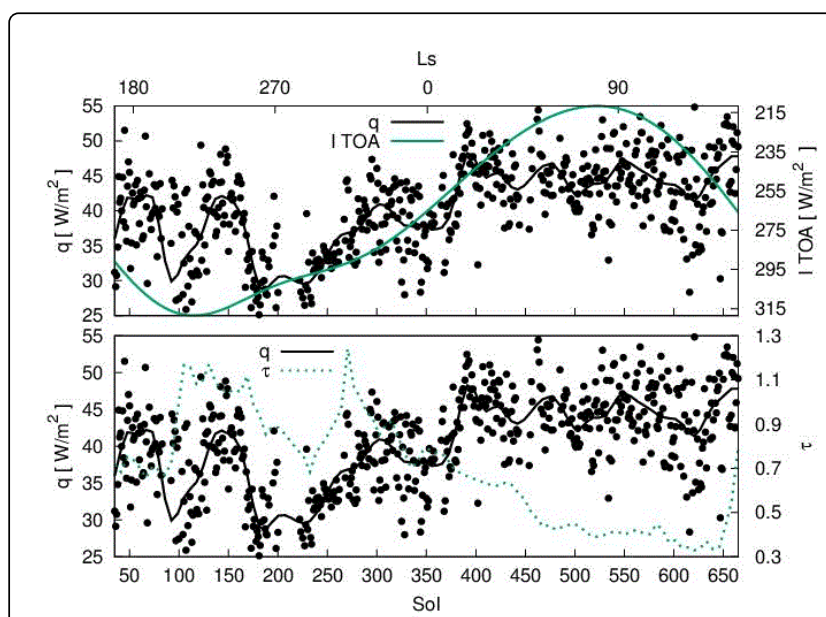

Figure 6: Annual variation of the daily solar insolation at TOA, the daily averaged heat-flux emitted by the rover (q), and the atmospheric opacity (produced by mineral aerosols in suspension). The plot below shows $\mathrm{q}$ throughout a martian year against the opacity of the atmosphere due to dust in suspension. The heat flux between the rover and the ground varies seasonally between 10 and $22 \mathrm{Wm}^{-2}$.

In the second step, the thermal contamination of the ground around the rover was used as a reference to quantify the effect of this radiative heat-flux on the ground. When the rover changes positions, the soil is not contaminated initially so, observing the temperature evolution when it remains still on a new position after a shift, it is possible to infer, with relation to the different kind of ground the rover has gone over, the magnitude of the contamination induced by rover alone [10]. This analysis required the implementation of the routines splint and spline to compare measurements at different days and times. An example is shown in Figure 7, where the period of time when the rover moved is shadowed. Similar difference curves have been calculated for other sols, (Figure 8). The temperature difference

$$
\begin{aligned}
& S_{a v}\left(L_{s}, \theta\right)=\frac{S_{o}}{\pi} \cdot r_{a v r}(L s)^{2} \cdot\left[\cos (\delta(L s)) \cdot \cos (\theta) \cdot \sin (H(L s, \theta))+\frac{H(L s, \theta)}{180} \cdot \pi \cdot \sin (\delta(L s)) \cdot \sin (\theta)\right] \\
& \delta(L s)=\operatorname{asin}(\sin (\varepsilon) \cdot \sin (L s)) \\
& r_{a V r}(L s)=\frac{1+e \cdot \cos (L s-L s p)}{1-e^{2}} \\
& H(L s, \theta)=\operatorname{acos}(-\tan (\theta) \cdot \tan (\delta(L s)))
\end{aligned}
$$




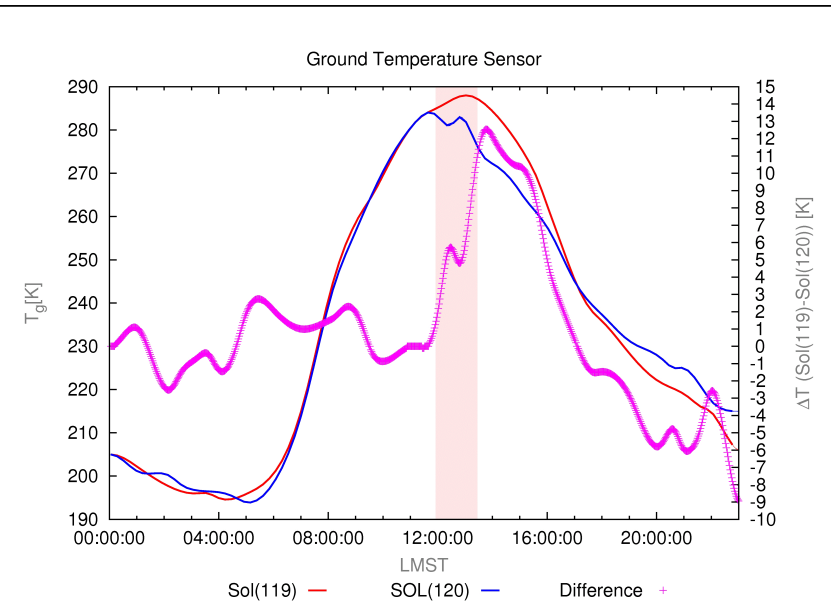

Figure 7: Interpolated functions for ground temperature values on sols 119 and 120. The pink curve shows the difference between them, and the period when the rover moved is marked with the shadowed bar.

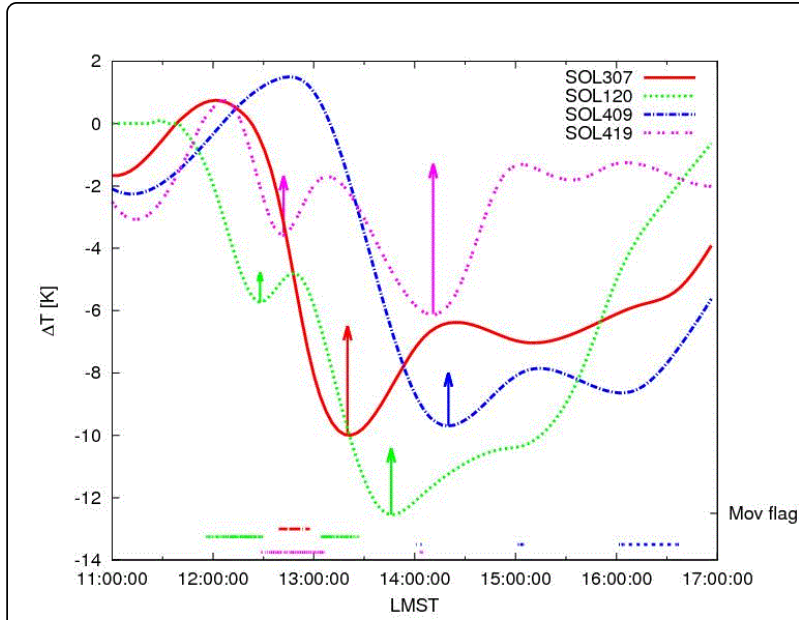

Figure 8: Differences of the ground temperature between the sols represented. The arrows show the total increase of the temperature after a shift of the rover.

\begin{tabular}{|c|c|c|c|c|c|}
\hline SOL & Ls [ ["] & $\begin{array}{l}\text { LMST (End of Movement) } \\
\text { [h:min] }\end{array}$ & $\Delta \mathrm{T}$ (End of Movement) $[\mathrm{K}]$ & $\Delta \mathrm{T}$ (Recovery) $[\mathrm{K}]$ & Heating Trend $[\mathrm{K} / \mathrm{min}]$ \\
\hline 120 & 220.50 & $12: 28$ & 6.8 & 1.0 & 0.048 \\
\hline 120 & 220.50 & $13: 46$ & 7.8 & 2.1 & 0.037 \\
\hline 307 & 336.91 & $13: 20$ & 10.7 & 3.6 & 0.065 \\
\hline 409 & 28.81 & $14: 20$ & 11.2 & 1.7 & 0.039 \\
\hline 419 & 33.51 & $12: 42$ & 4.3 & 1.8 & 0.069 \\
\hline 419 & 33.51 & 14:11 & 4.4 & 4.8 & 0.093 \\
\hline
\end{tabular}

Table 1: Summarize of rover induced contamination, sol of observation and LMST of the time when the rover moved. The rover induced thermal contamination ( $\Delta \mathrm{T}$ (End of Movement) $[\mathrm{K}])$ is calculated for each sol.

\section{Results and Highlights}

Besides having served to reinforce basic concepts of space and planetary physics and to introduce the students to the use of PDS, the training through this practical case has built a bridge between the academic formation and the real research work, overcoming the gap than often separates one sphere from the other.

As for the results, it has been concluded that the rover causes an increase of 4 to $11 \mathrm{~K}$ in the ground temperature values registered by the GTS. This rover-induced thermal perturbation produces a systematic positive offset of local temperatures, and should be taken into account when comparing with orbiter-based observations; i.e., the local Martian surface temperature should be about 4 to $11 \mathrm{~K}$ lower than the ones observed by the REMS GTS on board the MSL rover. Of course usual Earth weather stations are not mounted on hot moving platforms, thus acquiring environmental data on the MSL rover with its RTG power source is a challenge for REMS.

Regarding the behavior of the platform under different seasonal conditions, the evaluation of the radiative heat-flux has shown the seasonality of the Martian climate, the correspondent variations of temperature and radiation fluxes, and the strong influence of atmospheric dust on it. On the other hand, it has been found that during the south hemisphere autumn and winter season (Ls $0^{\circ}$ to $180^{\circ}$ ), the radiative-heat flux is higher, whereas during the south hemisphere spring and summer seasons ( $\mathrm{Ls} 180^{\circ}$ to $360^{\circ}$ ), the radiative flux is lower. In other words, there is a significant anti-correlation between TOA insolation and radiative loss of the rover. Furthermore, during the season of dust activity, when the atmosphere opacity increases, increments of atmospheric opacity seem to increase the radiative-heat flux from the rover to the ground. In summary the rover to ground radiative heat-flux shows a seasonal and environmental variability. Other effects, not considered here, such as winds and changes in the boundary layer pressure and air density, may affect the dissipation of heat from the rover and ground by other means such as wind produced convection and, to a much lesser extent, conduction to the air.

Furthermore, this training study has allowed to constraint the magnitude of the rover radiative heat flux between 10 and $22 \mathrm{~W} / \mathrm{m}^{2}$, which is more than $10 \%$ of the solar daily averaged insolation at the top of the atmosphere. The Sun action is the most important heat 
Page 7 of 8

source on Mars. During the day hours, the solar flux is responsible for increasing the surface temperature from the coldest night-time temperatures to the maximum day-temperature. It should be noticed that the mean amplitude of the thermal variation (day-to-night) of the ground surface at Gale crater during its first Martian year was of $76 \mathrm{~K}$, (Figure 9).

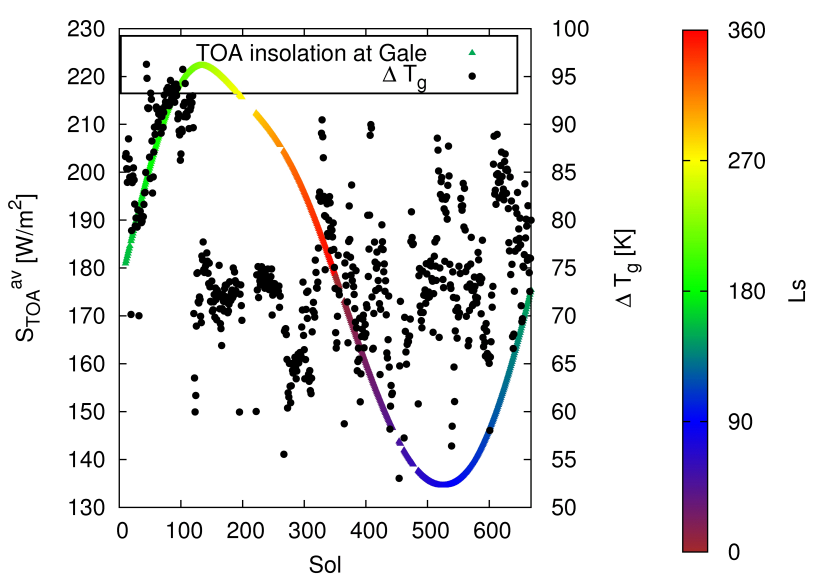

Figure 9: Diurnal thermal variation (day-to-nightdifference) of the ground surface at Gale crater during its first Martian year and comparison with the solar insolation at TOA. Ls is indicated in colour coding.

All these analyses have led finally to the finding of some novel discoveries regarding the thermal control of spacecraft that may be of interest for the design of future Mars surface exploration missions. Given that the radiative heat-flux losses of the rover are estimated to be about $10 \%$ of the solar flux, the order of magnitude of the ground temperature offset generated by the rover alone should be about $10 \%$ of this magnitude. This is compatible with the results summarized in Table 1, which on average estimate a rover induced contamination of $7.5 \mathrm{~K}$. This matter will have to be investigated in further detail for future surface missions such as Insight. The primary payload of the Insight lander consists of a seismometer and the Heat Flow and Physical Properties Package (HP3) [11], which will measure heat flow at the landing site in Elysium Planitia $\left(139^{\circ} \mathrm{E} 1^{\circ} \mathrm{N}\right)$. HP3 will emplace a suite of temperature sensors to a depth of up to $5 \mathrm{~m}$ into the Martian regolith and will monitor subsurface temperatures for a full Martian year. The seasonal variation of the annual thermal wave is expected to be removed by seasonally averaging [12]. The internal heat-flux to be measured is expected to be of the order $20 \mathrm{~mW} / \mathrm{m}^{2}$ which, according to the results presented above, are three orders of magnitude smaller than the MSL induced radiative heat-flux at the surface of the ground, and it is also three orders of magnitude smaller than its inter-annual variability. Given that the spacecraft-surface interaction induces a large seasonally varying radiative heat-flux, these effects have to be accurately investigated. Future pure research studies will incorporate CFD (Computational Fluid Dynamics) simulations of the MSL platform and surface under Martian atmospheric conditions. These studies shall incorporate the thermal information of the MSL platform from different components (RTG, rover sides and deck, wheels, mast and cameras, etc.). This shall be done to quantify in a more precise way the radiative heat flow of the rover side and rover RTG under diurnal (with solar heating) and night-time conditions. These analyses shall include the complex geometry of the platform, the existence of different thermal gradients within it, the conduction through the wheels, and the role of wind-forced convection, as well as atmospheric density changes, $\mathrm{CO}_{2}$ thermal conductivity changes with temperature, and changes in the atmospheric opacity. Similar simulations studies may be of interest for other Mars surface exploration platforms such as the Insight mission or the ExoMars rover and the NASA 2020 rover.

So, besides the contribution of the study to the training of future engineers and scientists and to the development of their skills and competence, this practical case shows that, given that the analysis of data from the missions in progress is increasingly complex, the constant revision can be highly useful for the development of the discipline, including the analysis performed by less specialized people as long as they are led adequately.

In the years to come, some others probes will come to intensify the study of Mars. Trace Gas Orbiter (TGO) and the Entry Descend Landing Demonstration Module (EDM) (ESA/Roscosmos) will launch in 2016, as well as the InSight lander by NASA. The ExoMars rover and surface platform, again by ESA/Roscosmos, will launch in 2020 together with a Curiosity's twin rover by NASA. The Indian Space Research Organization (ISRO) has scheduled a mission in the 2017-2020 timeframe, and the Japanese Aerospace Agency (JAXA) is considering a Mars Exploration Program that includes sending a surface rover mission (MELOS). The United Arab Emirates agency will start its activity with a mission to Mars in 2021, and China has planned as well a mission composed of an orbiter, a lander and a rover to be launched before 2020. Furthermore, Curiosity's energy source, RTG, has a foreseen lifespan of some 50 years. This means that the extended mission phase, which started on October the 1st 2014 after more than two years of operation on Mars, may thus be extended indefinitely for the time being, in which case, REMS shall provide the most extensive and complete environmental data set of groundatmosphere interaction on Mars by large, which will need a similarly extensive (and intensive) analytical effort from now on.

Therefore, it is convenient to implement strategies to extend the analytical work beyond the research teams involved, looking for support wherever it can be given, and assuring that each and every possible interpretation is considered. This work, which was originally defined as a practical study case pointed to education and as a user's guide to encourage the scientific and academic community to have access to the REMS PDS data and to take advantage of it for educational purposes, exemplifies how such a study can unveil unexpected and novel discoveries which are worth further investigation.

\section{References}

1. Gómez-Elvira J, Armiens C, Castañer L, Domínguez M, Genzer M, et al. (2012) The Environmental Sensor Suite for the Mars Science Laboratory Rover. Space Sci Rev 170: 583-640.

2. Gómez-Elvira J, Armiens C, Carrasco I, Genzer M, Gómez F, et al. (2014) Curiosity's Rover Environmental Monitoring Station: Overview of the First 100 Sols. Journal of Geophysical Research: Planets 119: 1680-1688.

3. Webster CR, Mahaffy PR, Atreya SK, Flesch GJ, Mischna MA, et al. (2015) Mars methane detection and variability at Gale crater. Science 347: 415-417.

4. Torres JM, Zorzano MP, Serrano PV, Harri AM, Genzer M, et al. (2015) Transient liquid water and water activity at Gale crater on Mars. Nature Geoscience 8: 357-361. 
Citation: Buenestado JF, Zorzano MP, Salinas AS, Méndez CF, Martín-Torres J (2015) Planetary Exploration; Mars on the Scope. Astrobiol

5. Golombek M, Grant J, Kipp D, Vasavada A, Kirk R, et al. (2012) Selection of the Mars Science Laboratory Landing Site. Space Science Reviews 170: 641-737.

6. Golombek MP, McSween HY (2014) Mars: Landing Site Geology, Mineralogy and Geochemistry. Encyclopedia of the Solar System 331-348.

7. Hamilton VE, Rafkin SCR, Pla-Garcia J, Sebastian E, Christensen PR, et al. (2014) Evening Surface Temperature Anomalies Observed by Curiosity in Gale Crater. Eighth International Conference on Mars Pasadena, California

8. Cucullu III GC, Daniel Zayas, Keith Novak, Pat Wu (2014) A Curious Year on Mars-Long-Term Thermal Trends for Mars Science Laboratory Rover's First Martian Year. 44th International Conference on Environmental Systems, Tucson, Arizona
9. Press WH, Teukolsky SA, Vetterling WT, Flannery BP (1996) Numerical Recipes in Fortran 90: $\quad$ The Art of Parallel Scientific Computing. (2ndedn), Cambridge University Press, Newyork, USA.

10. María-Paz Z, Javier MT, Claire N, Manuel de la T, Victoria H, et al. (2013) Ground calorimetric studies using the REMS ground temperature sensor and the Curiosity rover. EGU General Assembly, Vienna, Austria

11. Grott M, Plesa AC, Daubar I, Spohn T, Smrekar S, (2015) Retrieving the Martian Planetary Heat Flow from Measurements at Shallow Depth. 46th Lunar and Planetary Science Conference, The Woodlands, Texas

12. Grott M, Helbert J, Nadalini R (2007) Thermal structure of Martian soil and the measurability of the planetary heat flow. J Geophys Res 112: E9. 